\title{
Algoritmos como herramienta en la búsqueda de nuevos datos para la resolución de problemas sobre isometrías del plano'
}

\author{
Brigitte Johana Sánchez Robayo* \\ Jaime Fonseca González**
}

Artículo recibido: 15-01-2012 y aprobado: 15-11-2012
Resumen: Una vez reconocida la importancia que tienen los algoritmos en el proceso de resolución de problemas, particularmente en geometría, se identificaron teóricamente algunas posibles formas en las que se usan algoritmos que son conocidos para los resolutores, durante la solución de algún problema cuyo objeto central son las isometrías del plano. En este artículo se confirman, describen y evidencian los usos relacionados con la obtención de nueva información, y a partir del análisis de los datos, se sugiere una ampliación de la descripción de cada uso. Finalmente, se mencionan algunas cuestiones abiertas emergentes a partir de la realización del estudio y de la elaboración de conclusiones, también se citan algunas implicaciones del trabajo para la investigación y la didáctica de las matemáticas.

Palabras clave: Resolución de problemas, algoritmos, isometrías del plano.

\begin{abstract}
Algorithm as a tool for searching new dates to solve plane isometries problems
\end{abstract}

Abstract: Starting from the recognizing of the algorithms importance in problem solving process, particularly in geometry, some ways were identified in which using known algorithms by solvers during solving some problems whose central object is plane isometries. In this paper, some uses are described, confirmed and evidenced, these uses are linked obtaining new information up. Furthermore, the dates analysis is the support to suggest a description extended of each use. Finally, there are some open issues which have emerged from the carrying study and from making conclusions; also plenty of implications are mentioned to research and mathematics didactic fields.

Keywords: Solving problems, algorithms, plane isometries.

* Universidad Distrital Francisco José de Caldas: bjsanchezr@udistrital.edu.co

** Universidad Distrital Francisco José de Caldas: jaimejaimef@udistrital.edu.co

1 Una versión preliminar de este artículo ha sido publicada en las memorias del XX Encuentro de geometría y sus aplicaciones, Universidad Pedagógica Nacional, Bogotá, 23 a 25 de juni de 2011. 


\section{Introducción}

En Fonseca y Sánchez (2007) se estudiaron los procedimientos que emplean un profesor de matemáticas, un estudiante que se está preparando para ser profesor de matemáticas y un estudiante de educación Media, para resolver problemas sobre isometrías del plano, analizando específicamente, el uso que ellos le dan a algoritmos que reconocen para la solución de otros problemas.

Como resultado se encontraron diversos usos, clasificados en las siguientes dos categorías: solución del problema usando algoritmos para hallar datos y solución del problema usando algoritmos para determinar relaciones entre los elementos del problema. En este artículo se desarrollan los aspectos más relevantes de la primera categoría, tales como caracterización inicial y evidencias obtenidas a partir de la aplicación de dos cuestionarios que se validaron mediante pilotaje y revisión de expertos.

Cada cuestionario estuvo conformado por tres problemas, abordados por la población en diferentes momentos. El proceso de resolución de cada problema, fue registrado por medios audiovisuales, a la vez que los investigadores diligenciaban una rejilla diseñada de acuerdo con los usos de algoritmos propuestos. Adicionalmente en los problemas, cada uno de los integrantes de la población buscó diversas formas de solución, identificando en la verbalización del proceso, sus razonamientos y estrategias.

La identificación de los usos de algoritmos en la resolución de problemas proporciona herramientas específicas respecto a las estrategias que los resolutores consideran al momento de enfrentarse a un problema, por lo que se convierte en un instrumento potente al analizar estos procesos, tanto para aquellas personas interesadas en analizarlos desde cuestionamientos investigativos, como para el resolutor, al identificar nuevos posibles acercamientos a la resolución de determinado problema. Adicionalmente, a pesar de que los resultados de la investigación que aquí se presentan fueron obtenidos de la resolución de problemas sobre isometrías del plano, sugieren una extrapolación a la resolución de problemas en otras áreas de las matemáticas y, su estudio puede constituir un objeto de investigación para profesores interesados en la resolución de problemas como un elemento constitutivo de su hacer en el aula.

\section{Algoritmos y resolución de problemas}

Para proponer e identificar diversas formas en las que se pueden usar algoritmos al resolver problemas, fue necesario, en primera instancia, realizar una fundamentación sobre diversos aspectos relacionados con: resolución de problemas y algoritmos. Gracias a la documentación asociada al primero, se conceptualizaron diversos términos propios de esta acción y específicamente, los planteamientos de Polya permitieron validar una hipótesis, al aportar elementos que posibilitaron en la investigación, relacionar algoritmos como parte plausible de dicho hacer. Así mismo, las diferentes heurísticas que Polya propone y que de alguna forma son utilizadas por otros autores en la resolución de problemas, permitieron que como desarrollo propio de la investigación, se propusieran diversos usos de 
algoritmos que fueron relacionados con dichos procesos y que posteriormente fueron observados, analizados y, por tanto, validados mediante el estudio de caso. A continuación, se presentan los elementos teóricos más importantes sobre resolución de problemas, en los que además de especificar las definiciones asumidas, se muestran las relaciones establecidas entre algoritmos vs. resolución de problemas y uso de algoritmos vs. resolución de problemas.

La resolución de problemas ha sido una parte importante en la investigación en educación matemática desde los años sesenta, teniendo a Polya (1945) como uno de sus grandes precursores. Los trabajos desarrollados al respecto, han sido enmarcados por Shoenfeld (1985, citado por Alonso y Martínez, 2003, p. 82) en cuatro enfoques:

- Problemas que colocan la matemática en el contexto del "mundo real".

- Matemáticas aplicadas o modelos matemáticos: hace referencia al uso de matemáticas superiores en la solución de problemas aplicados al mundo real.

- Estudio de los procesos cognitivos: investigaciones referentes al estudio de aspectos del pensamiento matemático relacionados con la solución de problemas.

- Determinación y enseñanza de los tipos de habilidades necesarias para resolver problemas matemáticos.

A estos enfoques se adiciona la resolución de problemas vista como estrategia para la enseñanza y el aprendizaje de las matemáticas, que es quizás, el enfoque bajo el cual se desarrollan la mayoría de investigaciones actuales en educación matemática.

El trabajo de Polya (1945) puede en el cuarto enfoque y ha sido y continúa siendo, referente teórico para el desarrollo de investigaciones actuales sobre resolución de problemas (Anido y Rubio,1999; Barrosoy Gavilán, 2003). Además, dado que los intereses de la investigación no eran aspectos cognitivos, no consideraban las aplicaciones de las matemáticas ni una propuesta de enseñanza y estas no se concebían únicamente como una herramienta para describir el mundo real, el estudio se enmarcó en el cuarto enfoque de los propuestos por Shoenfeld y las herramientas teóricas fueron las planteadas en el trabajo de Polya (1945).

Por un lado, Polya (1945) plantea que el usar el término solución en diferentes frases puede generar confusiones en la forma de entenderlo, debido a que se usa para referirse: a las acciones realizadas al resolver un problema, al resultado de dichas acciones o al objeto que satisface las condiciones del problema. Por otro lado, Puig (1996) diferencia los términos resultado, solución y resolución, estableciendo que el primero es el dato que cumple las condiciones del problema, el segundo corresponde a la presentación final de los pasos que conducen al resultado y el tercero, a las acciones que se realizan en la búsqueda de la solución. Considerando las diferencias planteadas por Polya (1945) y Puig (1996), coincidimos con Codina y Rivera (2001) al concebir la solución como el objeto que cumple las condiciones dadas en el problema y la resolución como el conjunto 
de acciones que realiza el resolutor que lo pueden llevar a la solución del problema (por ejemplo, encontrar la incógnita).

Dentro de su propuesta, Polya (1945) sugiere cuatro fases para la resolución de un problema: comprender el problema, concebir un plan, ejecutar el plan y examinar la solución obtenida; si durante las dos últimas fases es posible perfeccionar, sistematizar y eximir de ambigüedades un conjunto finito de pasos que den solución al problema, se dice que este admite al menos un algoritmo de solución'.

De ser así, existe correspondencia entre las partes del problema (datos, condiciones e incógnita) y los componentes del algoritmo (datos iniciales, pasos y datos finales) respectivamente. Por ejemplo, en el problema de reflejar un punto $C$ del plano, a través de otro punto $\mathrm{X}$ del mismo plano (esto es $[\mathrm{X}](\mathrm{C})$, la reflexión de $\mathrm{C}$ por $\mathrm{X}$ ), se puede observar lo relacionado en la tabla 1.

\begin{tabular}{|c|c|}
\hline Problema & Algoritmo \\
\hline $\begin{array}{l}\text { Datos: } \\
\text { Puntos C y X, }[X]\end{array}$ & $\begin{array}{l}\text { Datos Iniciales: } \\
\text { Punto } C \text { y } X,[X]\end{array}$ \\
\hline $\begin{array}{l}\text { Condición: } \\
\text { el punto resultante debe } \\
\text { ser la reflexión de } C \text { a } \\
\text { través de }[X] \text {; debe ser } \\
\text { el punto } C^{\prime} \text { tal que } C \text {, } \\
\text { X y C' son colineales y } \\
\overline{C X} \cong \overline{X C}\end{array}$ & $\begin{array}{l}\text { Pasos de un algoritmo de } \\
\text { solución: } \\
\text { Trazar } \overleftrightarrow{\text { XC }} \\
\text { Trazar una circunferencia de } \\
\text { centro X y radio } \overline{X C} \text {. } \\
\text { La circunferencia construida } \\
\text { tiene dos puntos de intersección } \\
\text { con } \overleftrightarrow{\text { XC }: C \text { y } C} \\
\text { (que corresponde a la reflexión } \\
\text { de } C \text { a través de } \mathrm{X} \text { ). }\end{array}$ \\
\hline $\begin{array}{l}\text { Incógnita: } \\
{[X](C)=C}\end{array}$ & $\begin{array}{l}\text { Datos finales: } \\
{[X](C)=C^{\prime}}\end{array}$ \\
\hline
\end{tabular}

Tabla 1. Relación entre problema y algoritmo

1 Es de aclarar que Polya no define ni hace referencia a este término, sino que hace parte de la conceptualización realizada en la investigación.
Si existe un algoritmo de solución de un determinado problema (denominado problema auxiliar) que puede ser usado durante la resolución de otro, ya sea para encontrar más información de los datos o para encontrar nuevas relaciones entre ellos, entonces se dirá que se ha usado un algoritmo en la resolución del problema. Los dos propósitos que se acaban de mencionar determinanlas dos categorías que se consideraron durante la investigación y que permitieron clasificar y caracterizar usos de algoritmos. Sin embargo, este documento se centrará en los usos asociados a la primera categoría ${ }^{2}$ que se enuncian y describen a continuación.

\section{Solución del problema usando algoritmos para hallar datos}

En esta categoría se encuentran aquellos usos que se presentan cuando se emplean los algoritmos de solución de problemas conocidos para encontrar la incógnita o elementos adicionales que no pertenecen al problema inicial, pero que aportan información importante para su solución. Estos últimos son denominados elementos auxiliares.

Una revisión del trabajo de Polya condujo a proponer los siguientes usos de algoritmos, que se verificaron en la experimentación y cuya caracterización se amplió de acuerdo con el análisis elaborado en la aplicación de los cuestionarios:

- Solución inmediata del problema por medio de un algoritmo.

- Composición iterada de un mismo algoritmo.

2 Se puede encontrar información más detallada sobre esta clasificación en Fonseca y Sánchez (2010). 
- Composición de dos o más algoritmos diferentes.

- Uso de un algoritmo análogo.

- Uso de un algoritmo para determinar elementos auxiliares.

\section{Solución inmediata del problema por medio de un algoritmo}

Dentro de los procedimientos que propone Polya para resolver un problema se encuentra la generalización, que consiste en pasar del examen de un problema o un conjunto de problemas al examen de un conjunto más amplio de problemas que lo(s) contiene(n). El problema que consiste en solucionar todos los problemas del conjunto más amplio recibe el nombre de problema general, mientras que cada uno de los problemas de este conjunto recibe el nombre de problema particular.

Cuando el problema general admite un algoritmo de solución, éste puede usarse para resolver el problema particular (figura 1).

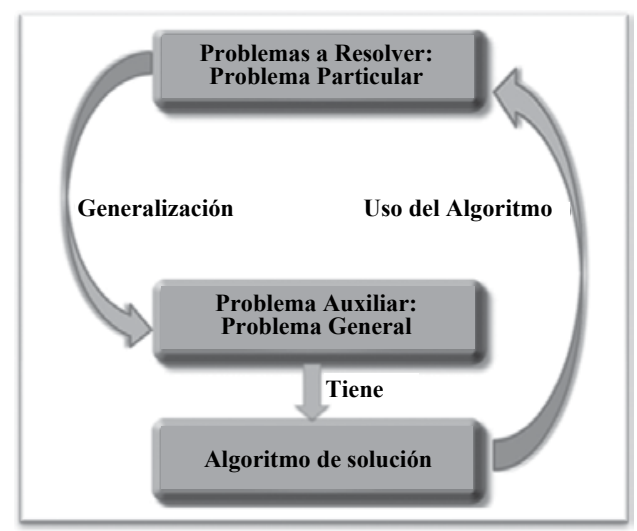

Figura 1. Esquema del uso de algoritmo para resolver un problema particular

En el caso en que se conozca un algoritmo de solución del problema general y se aplique sin mayores variaciones a los datos del problema particular dado, se dice que se ha realizado una solución inmediata del problema por medio de un algoritmo.

\section{Composición iterada de un mismo algoritmo y composición de dos o más algoritmos diferentes}

Otro de los procedimientos propuestos por Polya (1945) es la descomposición y recomposición, en los cuales se fija una idea directriz para la solución del problema y éste se divide de manera que el tratamiento se centre en los detalles del problema, cada uno de ellos se convierta en un problema auxiliar. Luego de solucionar cada uno de estos problemas auxiliares, la directriz antes fijada permitirá componer todas las soluciones y encontrar la solución del problema original. De hecho, es natural intentar descomponer el problema en otros conocidos, de modo que si éstos tienen un algoritmo de solución, su concatenación permita la solución del problema original o la construcción de un algoritmo que se use para su solución.

En el caso en el que se tome una parte o la totalidad de los algoritmos de solución de los problemas auxiliares, para encontrar la solución del problema original, se pueden presentar la composición iterada de un mismo algoritmo o la composición de dos o más algoritmos diferentes. El primer uso puede presentarse cuando: se utiliza repetidas veces un mismo algoritmo, los algoritmos de solución de los problemas auxiliares en los que se descompuso el problema original comparten la secuencia de pasos, o cuando los problemas auxiliares forman parte de un problema general cuya solución se puede obtener mediante la 
aplicación de un algoritmo. El segundo uso puede presentarse si se aplican dos o más algoritmos que dan solución a los problemas auxiliares en los que se descompuso el problema original, donde cada uno de los algoritmos difieren en al menos uno de sus pasos.

\section{Uso de un algoritmo análogo}

En la analogía, como otro de los procedimientos que propone Polya (1945), es posibleutilizar el método o el resultado obtenido de la solución de problemas análogos para solucionar el problema original $^{3}$. Específicamente, si se utiliza el algoritmo de solución de un problema análogo, ya sea repitiendo algunos pasos, variando únicamente los datos iniciales o tomando los datos finales como elementos auxiliares del problema original, se dirá que se usó un algoritmo análogo para hallar datos.

\section{Uso de un algoritmo para determinar elementos auxiliares}

En la resolución de un problema es necesario introducir elementos auxiliares con frecuencia, ya sea para ampliar la cantidad de datos, determinar nuevas relaciones, o simplemente comprender el problema; más aún, en geometría donde algunos de los elementos que se introducen se obtienen por construcción y la representación gráfica es frecuentemente empleada para comprender el problema. Por tanto, el uso de algoritmos para determinar elementos auxiliares se presenta cuando el elemento introducido es de vital importancia en la resolución del problema original.

3 Para profundizar sobre analogía, consultar Polya $(1945,1966)$.

\section{Algunas evidencias de los usos de algoritmos para hallar datos}

Los casos de usos de algoritmos que se presentarán se obtuvieron de la aplicación de dos problemas. El primero de ellos se muestra en la figura 2.

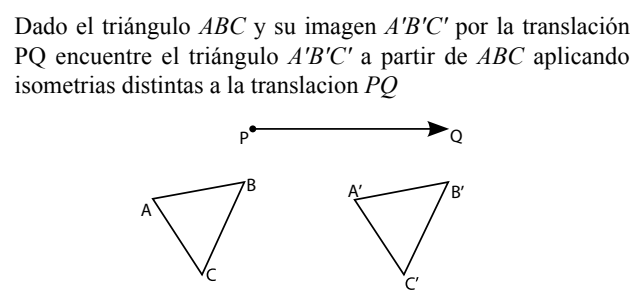

Figura 2. Problema aplicado

En la solución elaborada por el estudiante que se está formando como profesor, se observó que aquel asoció el problema con la construcción de dos reflexiones axiales que, aplicadas a un polígono, determinan una imagen equivalente a la de aplicar una traslación. Este último problema había sido abordado anteriormente por el estudiante y su algoritmo de solución se aplicó de manera directa, sin mayores variaciones a la solución de este problema, obteniendo la solución inmediata del problema por medio de un algoritmo. Esta inferencia se hace teniendo en cuenta la siguiente afirmación hecha por el estudiante durante la resolución del problema:

En este caso diría: pues como la más directa (se refiere a una solución de un problema similar) que yo vería en este caso, sería [...] dos simetrías axiales, más o menos perpendiculares a ésta $(\overleftrightarrow{\mathrm{PQ}})$, la primera perpendicular a ésta [señala $\overleftrightarrow{\mathrm{PQ}}$ ] por este punto (P o B) y bueno, procedo. [Transcripción de lo dicho por el estudiante al resolver el problema]. 
La solución obtenida fue:

- Construye la recta $l_{1}$ perpendicular a $\overleftrightarrow{\mathrm{PQ}}$ por $\mathrm{C}$.

- Refleja $\triangle \mathrm{ABC}$ por $\mathrm{l}_{1}$.

- Construye la mediatriz de $\overline{\mathrm{CC}}$ ' y la nombra $\mathrm{l}_{2}$.

- La composición $\left[\mathrm{l}_{2}\right]\left[\mathrm{l}_{1}\right](\triangle \mathrm{ABC})$ es $\triangle \mathrm{A}^{\prime} \mathrm{B}^{\prime} \mathrm{C}^{\prime}$.

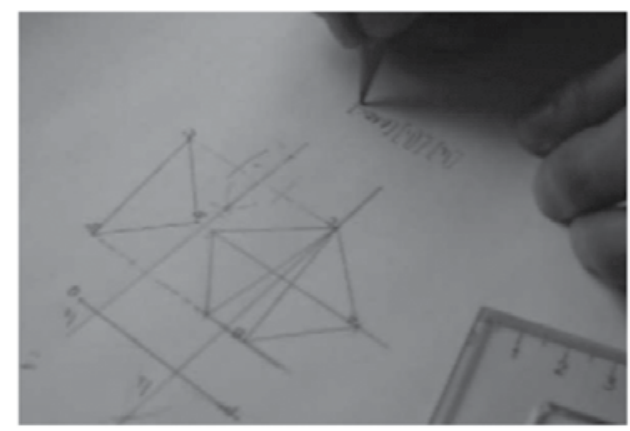

Figura 3. Evidencia de la solución inmediata del problema por medio de un algoritmo

Buscando una forma diferente de resolver el problema, el estudiante plantea la intención de usar únicamente rotaciones con centros convenientes, de modo que haga coincidir la imagen del $\triangle \mathrm{ABC}$ por $\left[\mathrm{l}_{1}\right]$ con $\triangle \mathrm{A}^{\prime} \mathrm{B}^{\prime} \mathrm{C}^{\prime}$. De esta forma, como lo describe durante el proceso de resolución, el estudiante da evidencias de su intención de usar el algoritmo de manera repetida, para realizar la rotación de un triángulo a través de un punto, con el fin de obtener otras dos transformaciones cuya imagen sea equivalente a la de una traslación, usando de esta manera la composición iterada de un mismo algoritmo. La transcripción de lo expresado por el estudiante se encuentra a continuación:

[...] la reflexión la puedo ver como una rotación. $Y$ después $l o$ vuelvo a rotar y vuelve a quedar en el mismo sitio pero más lejos, entonces estoy viendo en ésta [señala la gráfica de la primera solución], debe haber algún punto especial [indica $[\mathrm{h}](\triangle A B C)$ ] sobre el cual rotar, para convertir este, y aquí ya pasarlo al otro lado.

Este intento resulta fallido debido a que, según el estudiante, no encontró el centro de la rotación que le permitiera, solucionar el problema y en cambio, le estaba generando confusión. Esto era natural, puesto que en ningún caso la reflexión axial se puede expresar como una rotación (tal como él lo expresa).

Otro intento fallido consistió en aplicar una rotación y luego una traslación. Sin embargo, esta composición no le sirvió porque la aplicación de la rotación le mostraba un cambio en la dirección con respecto al $\Delta \mathrm{A}^{\prime} \mathrm{B}^{\prime} \mathrm{C}^{\prime}$.

Llegué a esa idea porque estaba pensando en rotación y después traslación, pero no me servía con rotación y después trasladar porque quedaba al revés, no quedaba; quedaba en otra posición, entonces pensé en otro movimiento y se me vino fue rotación, ieh! traslación. [Transcripción de lo dicho por el estudiante al resolver el problema].

La idea a la que el estudiante se refiere es la composición de traslaciones para resolver el problema. El intento anterior, evidencia el uso de composición de dos o más algoritmos diferentes y le permitió establecer una segunda forma de solucionar el problema: utilizar dos traslaciones de manera que su composición, sea, por tanto, usa composición iterada de un mismo algoritmo. 
La traslación la veo como, [...] podría hacer esta otra traslación (traslación que parte de $P$, diferente a $[P Q]$ y se visualiza en la figura 4$),[. .$.$] y después$ de aquí hasta acá a este punto [hace referencia a la traslación determinada por $Q$ diferente a y cuya representación la expresa con la escuadra como se visualiza en la Figura 4], y sería lo mismo trasladar el triángulo $(\triangle A B C)$ acá [señala la ubicación de la imagen] y pasarlo acá [señala la ubicación de $\left.\triangle A^{\prime} B^{\prime} C^{\prime}\right]$. Pues es algo. No sé. Me parece tonto. Esa sería como una solución, pero no estaría a la altura, y pues quiero hacer algo distinto a una traslación. [Transcripción de lo dicho por el estudiante al resolver el problema].

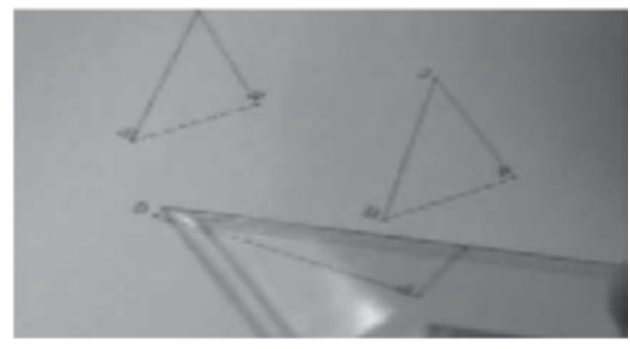

Figura 4. Evidencia de la composición de dos o más algoritmos diferentes

En esta intervención, el estudiante hace explícito su plan: descomponer el problema para realizar solo traslaciones, pues conoce algoritmos para realizarlas. El procedimiento de solución consiste en componer de manera iterada este mismo algoritmo y así encontrar otro que le permita solucionar el problema.

Una evidencia del uso de algoritmo para hallar elementos auxiliares fue obtenida en el proceso de resolución de este mismo problema, realizado por el profesor de matemáticas. En la búsqueda de una tercera solución, el estudiante expresó su interés en usar composición de diversas isometrías.

[...] Pensaba, de pronto, en otro movimiento que me permitiera obtener esa figura, pero tendría que hacer una composición de movimientos para obtener [quiere obtener $\triangle A^{\prime} B^{\prime} C^{\prime}$ ].

[...] Estoy pensando en si hago una reflexión central aquí [indica punto $C$ ] y podría obtener una, hacer una después, o sea, primero hacer una reflexión central teniendo como centro $C$ ¿sí? Obtenemos una figura y sí me daría, podría obtener esta figura (posible imagen de $\triangle A B C$ por [C]), también a partir de ahío sea, después hacer una reflexión axial. [Transcripción de lo dicho por el estudiante al resolver el problema].

Durante esa búsqueda, la imagen de $\triangle \mathrm{ABC}$ por [C] le permitió observar que la segunda isometría que debía usar no era una reflexión axial, sino una reflexión central; también, usó esta imagen y el triángulo A'B'C' para obtener el centro de la reflexión. Por tanto, la imagen de $\triangle \mathrm{ABC}$ por $[\mathrm{C}]$ fue un elemento auxiliar fundamental en la resolución del problema, obtenido por la aplicación del algoritmo de la reflexión central. Por ello, se usó un algoritmo para determinar elementos auxiliares.

Una evidencia del uso de un algoritmo análogo para hallar datos, se obtuvo de la aplicación del siguiente problema: 
Una solución propuesta por el estudiante que se está formando para profesor fue:

Dados tres puntos $A, B$ y $C$, considere un punto $X$ distinto de ellos. Si el punto $X$ tiene que ser reflejado por lo menos una vez por cada uno de los puntos $A, B y$ $C$, ¿qué isometrías se deben realizar para volver al mismo punto de partida? Las isometrías deben definirse a partir de los puntos A, $B$ o $C$, o de objetos construidos con base en tales puntos.

- Refleja X"' por C, obteniendo X".

- Refleja X” por B, obteniendo X'.

- Refleja X' por A, obteniendo X.

En la búsqueda de otra solución, el estudiante construyó mediatrices y medianas de $\triangle \mathrm{ABC}$ intentando encontrar alguna relación entre estas y datos del problema. Sin embargo, no tiene éxito alguno, por lo que cambia la estrategia y quiere variar el orden de aplicación de las reflexiones centrales consideradas en la anterior solución. Por tanto, usa el algoritmo de la solución anterior en la búsqueda de otra solución. Esto es, uso de un algoritmo análogo para hallar datos, específicamente para encontrar $X$. Este plan de solución del problema, se puede evidenciar en la siguiente intervención:

No, no me salió nada, por ninguna, por ningún punto especial.

Estoy pensando en este momento en otra solución, ya dejé a un lado lo que estaba pensando. Ahora estoy pensando en si influye el orden de las reflexiones que haga respecto a cada punto; si digo que hago respecto a A (reflejar $X^{\prime \prime \prime), ~ p r i m e r o ~ m e ~ q u e d a r i ́ a ~ e l ~}$ punto por acá [indica una aproximación de $\left.[A]\left(X^{\prime \prime \prime}\right)\right]$. [Transcripción de lo dicho por el estudiante al resolver el problema].

Como se puede observar, el estudiante que se está formando para profesor plantea explícitamente el cuestionamiento que se hace respecto a la imagen que podría obtener, al cambiar el orden de las reflexiones consideradas en la anterior solución.

\section{Consideraciones finales}

A partir del análisis realizado a los procesos de resolución de problemas de cada uno de los miembros de la muestra, se identificaron acciones en las que se evidenciaban los usos de algoritmos propuestos, ampliando la caracterización inicialmente planteada de cada uno de ellos.

Así, la solución inmediata del problema por medio de un algoritmo es el uso más frecuente que conscientemente se le suele atribuir a los algoritmos y se encuentra ligado al tipo de problema rutinario ${ }^{4}$. Aunque este uso no está relacionado con el tiempo empleado por el resolutor, puede ser un indicador de que conoce un problema auxiliar.

Este uso también se presenta cuando se descompone un problema en problemas auxiliares, donde, al menos uno de ellos es rutinario; en este caso, puede generarse la composición de algoritmos

4 Dentro de la tipología que identifica Polya, se encuentran los problemas rutinarios, que pueden resolverse sustituyendo datos en problemas ya resueltos o siguiendo paso a paso la secuencia de algún ejemplo. 
si existen algoritmos de solución para cada uno de los problemas auxiliares en que se descompuso el problema original. Por tanto, se puede inferir que los dos usos mencionados pueden estar ligados cuando se presenta este caso.

Adicionalmente, si todas las condiciones del problema auxiliar están contenidas en las del problema original y el uso de algoritmo análogo se genera debido a la analogía entre los problemas, entonces el uso de este algoritmo puede llegar a convertirse en la solución inmediata de un problema por medio de un algoritmo.

La composición iterada de un mismo algoritmo no se asume únicamente en el caso en que la solución esté expresada como aplicación de un mismo algoritmo, sino cuando hay una intensión del resolutor de resolver el problema por la iteración de un determinado algoritmo, sugerida por el contexto del problema o por algún problema auxiliar cuya solución se obtiene utilizando tal algoritmo. A su vez, no se puede decir que se ha dado este uso cuando hay una obligación de resolver el problema utilizando un mismo algoritmo.

Además de la caracterización inicial de la composición de dos o más algoritmos diferentes, este uso también puede darse cuando el resolutor obtiene, como estrategia para resolver un problema, la verificación de diversas composiciones de isometrías; por ejemplo, una reflexión central y una translación, una reflexión axial y una translación.

Este uso también ocurre cuando la estrategia del resolutor consiste en aplicar un algoritmo a un subconjunto de los datos del problema, para luego encontrar la manera de hacerlos coin- cidir con los que hacen falta, es decir, cuando se ve obligado a crear o buscar otro algoritmo para tal fin.

No se considera que este uso se presente cuando la solución está expresada como composición de algoritmos diferentes, sin tener claridad de las razones que llevaron a proponer tal composición, pues es natural que la solución de un problema con solución algorítmica sea por composición de algoritmos que permiten obtener elementos auxiliares y resolver problemas auxiliares necesarios para la resolución del problema original.

En el uso de un algoritmo análogo, el algoritmo de solución del problema análogo más sencillo se emplea para tomar los datos finales como nuevos datos del problema original o para obtener la solución misma del problema original. Una vez se tiene un algoritmo de solución de un problema, si éste se emplea en la búsqueda de nuevas soluciones del mismo problema, suele presentarse el uso de un algoritmo análogo, pues se consideran datos o pasos del algoritmo de las soluciones dadas anteriormente al problema.

Este recurso también se presenta cuando el resolutor tiene un algoritmo de solución de más de dos $\operatorname{pasos}^{5}$ del problema original y modifica el orden de algunos de ellos, sin hacerlo en orden inverso.

Aunque se encuentre la relación de analogía entre dos problemas, no se garantiza el uso de algoritmo análogo ya que uno de los problemas debe tener

5 Si el algoritmo tiene menos de tres pasos, el único orden que puede tomarse corresponde al orden inverso. 
algoritmo de solución y emplearse en la resolución del problema original.

El uso de algoritmos para determinar elementos auxiliares se ha delimitado solo a aquellos casos en que el elemento introducido es de vital importancia en la resolución del problema. Esto, puesto que con frecuencia en la resolución de un problema es necesario introducir elementos auxiliares, ya sea para ampliar la cantidad de datos, determinar nuevas relaciones, o simplemente comprender el problema; más aún en geometría donde algunos de los elementos que se introducen se obtienen por construcción y la representación gráfica es empleada con frecuencia para comprender el problema.

Este estudio también permitió confirmar la existencia de algoritmos en geometría, donde las construcciones son un caso particular de los mismos. Tal existencia fue reconocida por el profesor de matemáticas, el estudiante que se está formando para profesor de matemáticas y el estudiante de educación Media. Así mismo, ellos calificaron como importante el papel de los algoritmos al resolver problemas de matemáticas en general y concibieron los algoritmos en geometría como una construcción geométrica-como se consideró en el desarrollo del estudio- y como la secuencia de acciones asociadas a la resolución de los problemas.

Adicionalmente, en el estudio se propusieron usos de algoritmos para hallar datos y usos de algoritmos para encontrar relaciones entre los datos de un problema. El estudiante que se está preparando para ser profesor de matemáticas fue quién le dio prioridad al primer tipo de uso (que es el desarrollado en este escrito), al momento de resolver problemas sobre isometrías del plano.

En términos generales, puede establecerse que al estudiar los procesos enmarcados en la resolución de problemas, un factor que se ha de considerar son los algoritmos involucrados, así como el uso que se le dé a los mismos.

Particularmente, este estudio tiene implicaciones desde el punto de vista de la investigación y de la didáctica. La primera se enfoca en aquellos intereses hacia la resolución de problemas y los procesos que en ella se encuentran inmersos; por un lado, los usos de algoritmos proporcionan información importante sobre algunas estrategias que utiliza el resolutor cuando se enfrenta a un problema; por otro, la categorización de los usos de algoritmos, puede aplicarse en el estudio de resolución de problemas de otros campos de las matemáticas. La segunda, como parte del conocimiento profesional del profesor que puede ser utilizado para orientar los procesos de resolución de problemas de sus estudiantes de modo que se familiaricen en particular, con las estrategias en las que se emplean algoritmos de solución de problemas conocidos de la geometría o de otras áreas de las matemáticas.

\section{Referencias}

Alonso, I. y Martínez, H. (2003). La resolución de problemas matemáticos. Una caracterización histórica de su aplicación como vía eficaz para la enseñanza de la matemática. Revista pedagogía universitaria, 8(3), 81 - 88.

Anido, M. y Rubio, H. (1999). Un ejemplo de aprendizaje en el sentido de Polya. Revista Latinoamericana de Inves- 
tigación en Matemática Educativa, 2(2-3), 5-17.

Barroso, R. y Gavilán, J. (2003). Resolución de problemas de geometría con Cabri II. Revista Números, 54, 23-30. Recuperado el 8 de octubre de 2010 de: www.sinewton.org/numero/numero/54/articulo02.pdf

Codina, A. y Rivera, A. (2001). Hacia una instrucción basada en la resolución de problemas: los términos problema, solución y resolución. En: P. Gómez y L. Rico (eds.). Iniciación a la investigación en didáctica de la matemática. Homenaje al profesor Mauricio Castro (pp. 125-135). Granada: Universidad de Granada.
Fonseca, J. Sánchez, B. (2010). Algunas relaciones entre algoritmos y resolución de problemas. Revista Tecne, Episteme $y$ Didaxis : TED, 28, 73-87.

Fonseca, J.y Sánchez, B.J. (2007). Uso de algoritmos en la resolución de problemas sobre isometrías del plano. Un estudio de caso. Tesis de maestría. Bogotá: Universidad Pedagógica Nacional.

Polya, G. (1945). Cómo plantear y resolver problemas.Trad. de Julián Zagazagoitia. México, D.F.: Trillas.

Polya, G. (1966). Matemáticas y razonamiento plausible. Madrid: Tecnos

Puig, L. (1996). Elementos de resolución de problemas. Granada: Comares. 